\title{
Controllability of star-shaped networks of strings *
}

\section{René DÁGER, Enrique ZUAZUA}

Departamento de Matemática Aplicada, Universidad Complutense, 28040 Madrid, Spain

Courriels : rene_dager@mat.ucm.es, zuazua@eucmax.sim.ucm.es

\begin{abstract}
In this note we consider a star-shaped network of vibrating strings. The problem of controllability when one control acts on the junction point is considered. A simple proof is given that, in particular, does not use Ingham inequalities, of the fact that the set of reachable data is dense, whenever the lengths of the strings are mutually irrational. The proof is based on an observability inequality with suitable weights on the Fourier coefficients that is easily obtained as a consequence of the time-periodicity of the solutions. Those weights can be estimated under certain algebraicity conditions imposed on the lengths of the strings. This allows to proof exact controllability results in Sobolev spaces of appropriate order. Further results are also presented concerning the control from a free end and diffusion processes.
\end{abstract}

\section{Contrôlabilité de réseaux étoilés de cordes}

Résumé. On considère un réseau étoilé de cordes vibrantes contrôlé au nœud de jonction. Nous présentons une preuve élémentaire (qui, en particulier, ne nécessite pas de l'inégalité d'Ingham) du fait que lorsque les longueurs des cordes sont mutuellement irrationnelles, l'ensemble de données contrôlables est dense dans l'espace d'énergie naturel. La preuve donne une inégalité d'observabilité avec des poids explicites sur les coefficients de Fourier. Sous des hypothèses d'algébricité des longueurs cela entraîne la contrôlabilité dans un espace de Sobolev. Nous présentons aussi des conséquences sur la contrôlabilité lorsque le contrôle agit sur un extrême libre du réseau et des processus de diffusion.

\section{Version française abrégée}

On considère un réseau étoilé de $n$ cordes vibrantes contrôlé au point de jonction:

$$
\left\{\begin{array}{cl}
u_{i, t t}-u_{i, x x}=0 & \text { dans }\left(0, \ell_{\mathrm{i}}\right) \times \mathbb{R} \\
u_{i}(0, t)=v(t), \quad u_{i}\left(\ell_{i}, t\right)=0 & \text { pour } \mathbb{R} \\
u_{i}(x, 0)=u_{i}^{0}(x), u_{i, t}(x, 0)=u_{i}^{1}(x) & \text { dans }\left(0, \ell_{\mathrm{i}}\right)
\end{array}\right.
$$

pour $i=1, \ldots, n$. Dans (1), $u_{i}=u_{i}(x, t)$ décrit le déplacement vertical de la $i$-ème corde et $v=v(t)$ est le contrôle.

Étant donné $T>0$ on cherche à décrire l'espace de données initiales qui, moyennant un contrôle $v \in L^{2}(0, T)$, peuvent être rameneés à zéro au temps $t=T$.

On démontre le résultat suivant:

THÉORÈme. - On suppose que tous les quotients $\ell_{i}: \ell_{j}$ sont irrationnaux pour $i, j=1, \ldots, n$, $i \neq j$. Alors, l'ensemble de données contrôlables est dense dans l'espace $V^{0} \times V^{-1}$.

\footnotetext{
* This work has been partially supported by grants PB96-0663 of the DGES (Spain) and the EU TMR project
} "Homogenization and Multiple Scales". 
$S i$, en outre, tous les $\ell_{i}: \ell_{j}$ sont des nombres algébriques irrationnaux alors l'espace $V^{n-1+\varepsilon} \times$ $V^{n-2+\varepsilon}$ est contrôlable pour tout $\varepsilon>0$.

Enfin, si les longueurs $\ell_{i}$ sont linéairement indépendantes sur $\mathbb{Q}$ et tous les $\ell_{i}: \ell_{j}$ sont algébriques alors les espaces $V^{1+\varepsilon} \times V^{\varepsilon}$ et $W^{1+\varepsilon} \times W^{\varepsilon}$ sont contrôlables.

Ici, $V^{s}$ et $W^{s}$ dénotent, essentiellement, le produit des espaces de Sobolev $H^{s}\left(0, \ell_{i}\right)$ pour $i=$ $1, \ldots, n$, muni de conditions de trace convenables aux points $x=0, \ell_{i}$.

Ce problème a été analysé dans [1], [2] et [9] à l'aide de généralisations de l'inégalité d'Ingham. Dans [1] on donne une réponse complète au problème lorsque $n=2$. Le cas $n$ quelconque est traité dans [2] mais la caractérisation d'espaces de données contrôlables n'est pas complète.

Notre approche ne nécessitant pas des inégalités d'Ingham, est bien plus simple et donne des réponses définitives quelque soit $n$. On utilise HUM (J.-L. Lions, [6]) pour se ramener à une question d'observabilité pour le système sans contrôle et, dû à la $2 \ell_{i}$-pèriodicité en temps des solutions de l'équation des ondes dans l'intervalle $\left(0, \ell_{i}\right)$ avec des conditions aux limites de Dirichlet homogènes, on obtient une inégalité avec les poids $\left(\gamma_{i, k}\right)^{2}$ dans les coefficients de Fourier des solutions, où

$$
\gamma_{i, k}=\prod_{i \neq j} \sin \left(\frac{k \pi \ell_{j}}{\ell_{i}}\right)
$$

On observe ensuite que, lorsque les $\ell_{i}: \ell_{j}$ sont irrationnaux, tous les $\gamma_{i, k}$ sont non nulles. Cela entraîne la densité de l'espace des données contrôlables. Ensuite, sous les conditions d'algébricité additionnelles du Théorème, on prouve l'existence de constantes $C_{\varepsilon}>0$ telles que

$$
\gamma_{i, k} \geq C_{\varepsilon} k^{-(n-1+\varepsilon)}, \quad \forall k \in \mathbb{N}, i=1, \ldots, n
$$

et

$$
\gamma_{i, k} \geq C_{\varepsilon} k^{-(1+\varepsilon)}, \quad \forall k \in \mathbb{N}, i=1, \ldots, n,
$$

respectivement. Ceci permet d'assurér l'existence d'espaces de Sobolev où le système est contrôlable.

\section{Introduction}

We consider a network of $n$ elastic homogeneous strings that undergo transversal vibrations and are coupled at a common end in a star-shaped configuration. The displacement of the junction point may be controlled by some external action.

Let the functions $u_{i}:\left[0, \ell_{i}\right] \times \mathbb{R} \rightarrow \mathbb{R}, i=1, \ldots, n$, be the deformations of the strings of lengths $\ell_{i}$. Then, the motion of the network is described by the system

$$
\left\{\begin{array}{cl}
u_{i, t t}-u_{i, x x}=0 & \text { in }\left(0, \ell_{\mathrm{i}}\right) \times \mathbb{R} \\
u_{i}(0, t)=v(t), \quad u_{i}\left(\ell_{i}, t\right)=0 & \text { in } \mathbb{R} \\
u_{i}(x, 0)=u_{i}^{0}(x), \quad u_{i, t}(x, 0)=u_{i}^{1}(x) & \text { in }\left(0, \ell_{\mathrm{i}}\right)
\end{array}\right.
$$

for $i=1, \ldots, n$.

Note that this model can be also viewed as a system of $n$ independent strings controlled at one of their ends by means of a common function $v$, i.e., as a simultaneous control problem.

It is known that problem (2) is well posed for $u_{i}^{0} \in L^{2}\left(0, \ell_{i}\right), u_{i}^{1} \in H^{-1}\left(0, \ell_{i}\right)$ and $v \in L^{2}(0, T)$ (see, e.g., [6]). Our goal is to drive the network to rest in finite time. That is, given initial states $u_{i}^{0}(x), u_{i}^{1}(x)$ of the strings and $T>0$, to choose the control function $v \in L^{2}(0, T)$ such that the solutions $u_{i}$ of $(2)$ verify

$$
u_{i}(x, T)=u_{i, t}(x, T)=0 .
$$


When this is possible, we shall say that the initial data $u_{i}^{0}, u_{i}^{1}$ are controllable in time $T$.

In order to give a precise sense to this problem, we introduce for $s \in \mathbb{R}$ the Hilbert spaces

$$
V_{i}^{s}=\left\{\varphi=\sum_{k \geq 1} a_{k} \theta_{i, k} ; \quad a_{k} \in \mathbb{R}, \quad\|\varphi\|_{s}^{2}=\sum_{k \geq 1} a_{k}^{2} k^{2 s}<\infty\right\}
$$

endowed with the norms \|\|$_{s}$, where $\left\{\theta_{i, k}\right\}_{k>1}$ is the orthonormal basis of $L^{2}\left(0, \ell_{i}\right)$ defined by $\theta_{i, k}(x)=\frac{2}{\ell_{i}} \sin \frac{k \pi x}{\ell_{i}}$, and $V^{s}=\prod_{i=1}^{n} V_{i}^{s}$ with the natural product structure. By $\bar{\varphi}$ we shall denote the vector with components $\varphi_{i}, i=1, \ldots, n$. Note that $V_{i}^{0}=L^{2}\left(0, \ell_{i}\right)$ and $V_{i}^{1}=H_{0}^{1}\left(0, \ell_{i}\right)$.

This problem has been studied in [1,9] for $n=2$ and in [2] for arbitrary $n$. In [9] it was shown that for any values of the lengths of the strings and any $s<1$ there exist initial data $u_{i}^{0} \in V_{i}^{s}$, $u_{i}^{1} \in V_{i}^{s-1}$ which are not controllable. In particular, the natural energy space $V^{0} \times V^{-1}$ of (2) is never controllable. In addition, when the strings have rationally-dependent lengths, the set of controllable initial data is not dense in $V^{0} \times V^{-1}$.

In $[1,9]$ necessary and sufficient conditions are also given to ensure that the controllability holds for $\bar{u}^{0} \in V^{1}, \bar{u}^{1} \in V^{0}$ for $n=2$, while in [2] sufficient conditions are given for the controllability of the spaces $V^{n-1+\varepsilon} \times V^{n-2+\varepsilon}$ with $\varepsilon>0$. In all these papers the results have been obtained resorting to new generalizations of the classical Ingham inequality for sums of complex exponentials. However, a complete description of the set of controllable data is not given in the cited papers when $n \geq 3$.

In this Note, we present a fairly simple idea that allows to obtain weighted observability inequalities. This provides necessary and sufficient conditions for the approximate controllability (i.e., for the existence of a set of controllable data which is dense in $V^{0} \times V^{-1}$ ) to hold in the minimal time $T_{0}=2 \sum_{i=1}^{n} \ell_{i}$ and allows to characterize spaces of controllable data. These results may be easily extended to the case when the control acts on one of the free extremes of the network and then for general tree-shaped networks. This latter problem will be considered elsewhere.

\section{Main results}

A direct application of the Hilbert Uniqueness Method of J.-L. Lions (see [6]) shows that all the initial data $\bar{u}^{0} \in V^{s}, \bar{u}^{1} \in V^{s-1}$ are controllable if, and only if, there exists a constant $C>0$ such that the observability inequalities

$$
C \int_{0}^{T_{0}}\left|\sum_{j=1}^{n} \varphi_{j, x}(0, t)\right|^{2} d t \geq\left\|\varphi_{i}(x, 0)\right\|_{1-s}^{2}+\left\|\varphi_{i, t}(x, 0)\right\|_{-s}^{2}
$$

are verified by the solutions of the adjoint problem

$$
\left\{\begin{array}{cl}
\varphi_{i, t t}-\varphi_{i, x x}=0 & \text { in }\left(0, \ell_{\mathrm{i}}\right) \times \mathbb{R} \\
\varphi_{i}(0, t)=\varphi_{i}\left(\ell_{i}, t\right)=0 & \text { in } \mathbb{R}
\end{array}\right.
$$

for every $i=1, \ldots, n$.

The following theorem is a crucial technical step. It provides weighted observability inequalities for the solutions of (4).

TheOREM - Let $\bar{\varphi}^{0} \in V^{1}, \bar{\varphi}^{-1} \in V^{0}$ and $\left\{a_{i, k}\right\}_{k \geq 1},\left\{b_{i, k}\right\}_{k \geq 1}$ be the Fourier coefficients of $\varphi_{i}^{0}$, $\varphi_{i}^{1}$ respectively, in the bases $\left\{\theta_{i, k}\right\}_{k \geq 1}$. Then, for every $i=1, \ldots, n$, the solution of (4) with initial data $\bar{\varphi}^{0}, \bar{\varphi}^{1}$ satisfies

$$
\int_{0}^{T_{0}}\left|\sum_{i=1}^{n} \varphi_{i, x}(0, t)\right|^{2} d t \geq 2 \sum_{k \geq 1}\left(\gamma_{i, k}\right)^{2}\left(\left(\frac{k \pi}{\ell_{i}}\right)^{2} a_{i, k}^{2}+b_{i, k}^{2}\right),
$$


where $\gamma_{i, k}=\prod_{j \neq i} \sin \frac{k \pi \ell_{j}}{\ell_{i}}$ and $T_{0}=2 \sum_{i=1}^{n} \ell_{i}$.

Proof. Note that, by density, it suffices to prove the assertion for $\bar{\varphi}^{0} \in V^{2}, \bar{\varphi}^{1} \in V^{1}$. Given a real number $a$ we shall denote by $\hat{a}$ the operator acting on a time-dependent function $f$ by $\hat{a} f(t)=\frac{1}{2}[f(t+a)-f(t-a)]$. Fix $i$. Due to the $2 \ell_{i}$-periodicity in time of $\varphi_{i, x}$, it holds that $\widehat{\ell}_{i} \varphi_{i, x}=0$.

If we define the operator $D_{i}$ as the composition of all the operators $\widehat{\ell}_{j}$ with $i \neq j$, then

$$
\int_{0}^{T_{0}}\left|\sum_{i=1}^{n} \varphi_{i, x}(0, t)\right|^{2} d t \geq \int_{\frac{1}{2} T_{0}-\ell_{i}}^{\frac{1}{2} T_{0}+\ell_{i}}\left|D_{i} \sum_{i=1}^{n} \varphi_{i, x}(0, t)\right|^{2} d t=\int_{\frac{1}{2} T_{0}-\ell_{i}}^{\frac{1}{2} T_{0}+\ell_{i}}\left|\sum_{i=1}^{n} D_{i} \varphi_{i, x}(0, t)\right|^{2} d t
$$

Now set $\psi=D_{i} \varphi_{i}$. This function is a solution of the wave equation $\psi_{t t}-\psi_{x x}=0$ in $\left(0, \ell_{i}\right) \times \mathbb{R}$ and $\psi(0, t)=\psi\left(\ell_{i}, t\right)=0$ for $t \in \mathbb{R}$. So, it is easy to see by developing $\psi$ into Fourier series that

$$
\int_{\alpha-\ell_{i}}^{\alpha+\ell_{i}}\left|\psi_{x}(0, t)\right|^{2} d t=4 E_{\psi}
$$

for any real number $\alpha$, where $E_{\psi}$ is the energy of $\psi$. On the other hand, as $\psi=D_{i} \varphi_{i}, E_{\psi}$ is given by

$$
E_{\psi}=\frac{1}{2} \sum_{k \geq 1}\left(\gamma_{i, k}\right)^{2}\left(\left(\frac{k \pi}{\ell_{i}}\right)^{2} a_{i, k}^{2}+b_{i, k}^{2}\right)
$$

and

$$
\psi_{x}(0, t)=D_{i} \varphi_{i, x}(0, t) .
$$

In view of (7), (8) and (9), inequality (5) holds from (6).

COROLLARY 1. - If for every $j \neq i$ the ratios $\ell_{i}: \ell_{j}$ are irrational numbers, all the coefficients $\gamma_{i, k}$ are different from zero and then

a) (uniqueness) The equalities $\sum_{i=1}^{n} \varphi_{i, x}(0, t)=0$ a.e. in $\left(0, T_{0}\right)$ imply $\phi_{i}^{0}=\phi_{i}^{1}=0$ for every $i$.

b) (approximate controllability) The set of controllable initial data of (2) in time $T_{0}$ is dense in $V^{0} \times V^{-1}$.

Remark 1. - Note that Corollary 2, which is a consequence of the observability inequality (5), does not guarantee the controllability in any Sobolev-type space $V^{s} \times V^{s-1}$. In fact, there are cases when, even though approximate controllability holds, there is no $s \in \mathbb{R}$ such that all the initial data from $V^{s} \times V^{s-1}$ are controllable. To see this, it suffices to consider the case of $n=2$ and to choose $\ell_{1}$ and $\ell_{2}$ so that $\ell_{1}: \ell_{2}$ is a Liouville's number. In this case, for any real $s$, the inequality $\left|\frac{\ell_{1}}{\ell_{2}}-\frac{p}{q}\right|<q^{-s}$ with $p \in \mathbb{N}$ holds for an infinite number of values of $q$. Then, if $\left\{\lambda_{k}\right\}_{k \geq 1}$ is the increasing sequence formed by the squared roots of the eigenvalues of both strings (i.e., by the numbers $\frac{p \pi}{\ell_{1}}, \frac{q \pi}{\ell_{2}}, p, q \in \mathbb{N}$ ) for every real $s$ there is a subsequence along which $\lambda_{k+1}-\lambda_{k}<\lambda_{k}^{-s}$. Therefore, inequality (3) is impossible for any $s$.

The key element of the previous example is that $\ell_{1}: \ell_{2}$ was chosen to be well-approximated by rationals. In fact, if this situation is avoided, then it is possible to give additional information on the weights $\gamma_{i, k}$ and then, to obtain positive results concerning the controllability in Sobolev-type spaces as the following proposition shows.

Proposition. - Let $\varepsilon>0$. Then

a) If the lengths $\ell_{i}$ verify

(A) - for every pair $i, j, i \neq j$, the ratios $\ell_{i}: \ell_{j}$ are algebraic irrational numbers, 
then there exits a constant $C_{\varepsilon}>0$ such that $\gamma_{i, k} \geq C_{\varepsilon} k^{-(n-1+\varepsilon)}$ for $k \in \mathbb{N}, i=1, \ldots, n$.

b) If the lengths verify

(B) - they are linearly independent over the field $\mathbb{Q}$ and, when $i \neq j$, the ratios $\ell_{i}: \ell_{j}$ are algebraic,

then there exists a constant $C_{\varepsilon}>0$ such that $\gamma_{i, k} \geq C_{\varepsilon} k^{-(1+\varepsilon)}$ for $k \in \mathbb{N}, i=1, \ldots, n$.

The first assertion follows from a Roth's theorem, but the algebraicity condition of the ratios $\ell_{i}: \ell_{j}$ may be replaced by a much weaker one. Indeed, a) still holds, if the ratios $\ell_{i}: \ell_{j}$ belong to a set $B_{\varepsilon}$ having full Lebesgue measure (see [3], pp. 104, 120). Assertion b) is a consequence of the Schmidt's theorem [8] on simultaneous rational approximation.

As an immediate consequence of this Proposition the following holds:

Corollary 2. - Let $\varepsilon>0$. Then

a) If the lengths of the strings of the network verify (A) all the initial data $u_{i}^{0} \in V_{i}^{n-1+\varepsilon}$, $u_{i}^{1} \in V_{i}^{n-2+\varepsilon}$ are controllable in time $T_{0}$.

b) If the lengths of the strings of the network verify $(\mathrm{B})$ all the initial data $u_{i}^{0} \in V_{i}^{1+\varepsilon}, u_{i}^{1} \in V_{i}^{\varepsilon}$ are controllable in time $T_{0}$.

Remark 2. - As it has been pointed out in $[2,4]$, the control time $T_{0}$ provided by Corollary 2 is the optimal one in the sense that the approximate controllability is impossible in any time $T<T_{0}$. Indeed, for every $T<T_{0}$, it is possible to construct explicit examples of non-zero smooth solutions of (4) such that $\sum_{i=1}^{n} \varphi_{i, x}(0, t)=0$ for $t \in[0, T]$.

Now define for $s \in \mathbb{R}$ the Hilbert spaces

$$
W^{s}=\left\{\bar{\varphi}=\sum_{k \geq 1} a_{k} \bar{\zeta}_{k} ; a_{k} \in \mathbb{R}, \quad\|\bar{\varphi}\|_{s}^{2}=\sum_{k \geq 1} a_{k}^{2} \omega_{k}^{2 s}<\infty\right\}
$$

where $\left\{\bar{\zeta}_{k}\right\}_{k \geq 1}$ is the orthonormal basis of $W^{0}=\prod_{i=1}^{n} L^{2}\left(0, \ell_{i}\right)$ formed by the eigenfunctions of the problem

$$
\left\{\begin{array}{cc}
z_{i, x x}=\lambda z_{i} & \text { in }\left(0, \ell_{\mathrm{i}}\right) \\
z_{i}(0)=z_{j}(0), & z_{i}\left(\ell_{i}\right)=0 \\
\sum_{i=1}^{n} z_{i, x}(0)=0, &
\end{array}\right.
$$

for $i, j=1, \ldots, n$, and $\omega_{k}$ are the corresponding eigenfrequencies of (10).

Following the scheme described in (4), it is possible to show that, under conditions (B), all the initial data $\bar{u}^{0} \in W^{n}, \bar{u}^{1} \in W^{n-1}$ are controllable in time $T_{0}$.

On the other hand, if $u \in W^{s}$, then $u$ may be expressed as

$$
u=v+w
$$

where $v \in V^{s}$ and $w \in W^{n}$. Consequently,

COROLlaRY 3. - If the lengths of the strings of the network verify $(\mathrm{B})$ then the spaces $W^{1+\varepsilon} \times W^{\varepsilon}$ are controllable in time $T_{0}$ for any $\varepsilon>0$.

Remark 3. - When $0<s<\frac{1}{2}$, it holds that $V^{s}=W^{s}$ and $V^{1+s} \subset W^{1+s}$. So, in this case, the space of controllable data given by Corollary 3 is strictly larger than that of Corollary 2 .

\section{Related problems and further results}

It is possible to obtain similar results for some related problems. 
a) The results above hold also when the control function is a force acting on the junction point or even when it is applied to the free end of one of the strings. For the latter problem results similar to those of $[4,5]$ may be obtained.

b) Following Russell's argument (see [7]) that guarantees the null controllability of the heat equation in any time $T>0$ when the wave equation is controllable for some $T>0$, under assumptions (A) or (B), the system of 1-d parabolic equations with a common boundary control

$$
\left\{\begin{array}{cl}
u_{i, t}-u_{i, x x}=0 & \text { in }\left(0, \ell_{\mathrm{i}}\right) \times \mathbb{R} \\
u_{i}(0, t)=v(t), \quad u_{i}\left(\ell_{i}, t\right)=0 & \text { in } \mathbb{R} \\
u_{i}(x, 0)=u_{i}^{0}(x), & \text { in }\left(0, \ell_{\mathrm{i}}\right)
\end{array}\right.
$$

may be shown to be controllable to zero for any $u_{i}^{0} \in L^{2}\left(0, \ell_{i}\right), i=1, \ldots, n$, in any time $T>0$. The same happens when the control is applied to the free end of one of the "strings".

c) Performing a coordinate transformation, the results given above extend to the just apparently more general situation where the equations in (1) are replaced by

$$
\frac{1}{a_{i}} u_{t t}-\left(a_{i} u_{i, x}\right)_{x}=0
$$

with $a_{i}$ being strictly positive smooth functions depending only on $x$. In this case the lengths $\ell_{i}$ should be replaced by the optical lengths $\tilde{\ell}_{i}=\int_{0}^{\ell_{i}} \frac{1}{a_{i}(\tau)} d \tau$. In particular, the results hold for strings having different constant densities.

\section{References}

[1] Avdonin S.A., Tucsnak M., Simultaneous controllability in sharp time for two elastic strings, ESAIM:COCV, to appear.

[2] Baiochi C., Komornik V., Loreti P., Ingham-Beurling type theorems with weakened gap conditions, to appear.

[3] Cassels J.M., An introduction to diophantine approximation, Cambridge Univ. Press, Cambridge, 1966.

[4] Dáger R., Zuazua E., Controllability of star-shaped networks of strings, In: Bermúdez A. et al. (Eds), Fifth International Conference on Mathematical and Numerical Aspects of Wave Propagation, SIAM Proceedings, 2000.

[5] Leugering G., Zuazua E., Exact controllability of generic trees, In: "Control of Systems Governed by Partial Differential Equations", Nancy, France, March 1999. ES AIM Proceedings.

[6] Lions J.-L., Contrôlabilité Exacte Perturbations et Stabilisations de Systèmes Distribués, Volume 1, Masson, Paris, 1988.

[7] Russell D.L., A unified boundary controllability theory for hyperbolic and parabolic partial differential equations, Studies in Appl. Math., 52(1973), 189-221.

[8] Schmidt W.M., Simultaneous approximation to algebraic numbers by rationals, Acta Math., 125(1970), 189-202.

[9] Tucsnak M., Weiss G., Simultaneous exact controllability and some applications, SIAM J. Cont. Optim., 38(2000), 1408-1427. 